\title{
Imprecisión de parámetros eritrocitarios con las reglas de 3 inversas según las guías CLSI H26-A2
}

Imprecision of erythrocyte parameters with rule 3 according to guidelines CLSI H26-A2

Jeel J. Moya Salazar', Liz G. Pio Dávila

\section{RESUMEN}

Objetivo: Determinar la imprecisión de parámetros eritrocitarios (Hematocrito, Hemoglobina y Recuento eritroide) con la regla de 3 según las guías del Instituto de Estándares Clínicos y de Laboratorio (CLSI) H26-A2, y discriminarlos por género. Métodos: Investigación cuasi experimental cualitativa, de corte transversal prospectiva. Se evaluó la calidad de la microcentrífuga y los capilares de acuerdo a la guía del Instituto de Estándares Clínicos y de Laboratorio CLSI H07-A3, para la determinación de microhematocrito manual se utilizó el método de D'angelo bajo la normatividad de la guía CLSI H26-A2, y las regla de 3 inversa. El análisis estadístico fue mediante SPSS versión 20.0. Resultados: De 156 muestras evaluadas mediante microhematocrito manual la imprecisión de Hematocrito, Hemoglobina y Recuento eritroide fueron $1,9 \%, 5,6 \%$ y $16,9 \%$, respectivamente $(p=<0,05)$.Los valores estimados a partir de Hematocrito superan todos los límites establecidos por el CLSI (fuera del rango máximo permisible de imprecisión intraensayo y de los límites de máxima variación establecidos por los requerimientos médicos). No hubo diferencias significativas de imprecisión entre género. Conclusiones: El porcentaje de imprecisión de los parámetros eritrocitarios con las reglas de 3 inversas es elevada principalmente para Hemoglobina y Recuento eritroide. Debiéndose descontinuar su uso o ajustar sus límites de error permisibles.

Palabras clave: Parámetros eritrocitarios, hematocito, imprecisión, control de calidad, hematología.

\section{ABSTRACT}

Objective: Determine the imprecision of erythrocyte parameters (hematocrit, Hemoglobin and Count Erythroid) to Rule 3 according to CLSI H26-A2 guides, and discriminate by gender. Methods: Qualitative Research quasi-experimental, prospective cross section. Microcentrifuge quality and capillaries was assessed according to the CLSI guide H07-A3, for determining manually microhematocrit D'Angelo method under the standards of the guide CLSI H26-A2, and the inverse rule of 3 was used. Statistical analysis was performed using SPSS version 20.0. Results: Of 156 samples tested by manual microhaematocrit the imprecision of hematocrit, Hemoglobin and Count Erythroid were $1.9 \%, 5.6 \%$ and $16.9 \%$, respectively $(p=<0.05)$. The estimated from hematocrit values exceed all limits established by the CLSI (outside the allowable maximum range of intraassay imprecision and maximum variation limits established by the medical requirements). There were no significant differences between genders imprecision. Conclusion: The percentage of imprecision erythrocyte parameters with rules 3 reverse is high mainly for Hemoglobin and Count Erythroid. Should be discontinue use or adjust limits on permissible error.

Keywords: Erythrocyte parameters, hematocrit, imprecision, quality control, hematology. 


\section{INTRODUCCIÓN}

El campo de la hematología siempre ha tenido un gran interés en el mundo en desarrollo, ha facilitado no sólo mejoras en la atención básica, sino que también han contribuido a una comprensión cada vez mayor de la ciencia detrás de los trastornos hematológicos. Esta ciencia se ha transformado radicalmente en las últimas décadas, con el advenimiento de la proteómica y la aplicación de la biología molecular (Cooper, Hart, Bates y Cotter, 2011).

A medida que el uso de técnicas diagnósticas de laboratorio en hematología se ha incrementado, se hizo evidente que los métodos de referencia estandarizados y controles estables eran esenciales para que los resultados obtenidos en diferentes laboratorios puedan ser comparables y se mantengan valores hematológicos los más cercanos a la realidad. A la fecha, todos los procedimientos en Hematología son evaluados dentro de un sistema de gestión de calidad (Nevalainen et al., 2000).Actualmente los procedimientos de evaluación de calidad son los siguientes: cianometahemoglobina para determinaciones Hemoglobina ( $\mathrm{Hb}$ ); Recuento de partículas para Eritrocitos (RBC) y glóbulos blancos (WBC) (Impedancia eléctrica, láser, conteo manual por hematimetría); WBC diferenciales (diff) por microscopia; conteos de plaquetas (PLT) por microscopia de fase y/o anti-CD61 y anti-CD41;Reticulocitos por citometría de flujo que distinga RBC; y volumen de células empaquetadas ( $\mathrm{PCV}=$ Packed Cell Volume) para las determinaciones de Hematocrito (Hto) (CLSI H26-A2, 2010; CLSI H20-A2, 2007; CLSI H07-A3, 2000).

El Instituto de Estándares Clínicos y de Laboratorio (CLSI) desarrolla sistemas de calidad para una mejor práctica clínica en salud y de laboratorio. Esta organización sin fines de lucro ofrece materiales de control y guías para el establecimiento de la calidad en el laboratorio clínico, sin embargo, es difícil y costoso obtener materiales de referencia sobre todo en países con bajo y mediano grado de desarrollo. En los países de América Latina hay muchos laboratorios que no cuentan con sistemas apropiados de evaluación y control de la calidad, por ello, la Organización Panamericana de la Salud patrocinó un curso de control de la calidad donde se produjeron y evaluaron materiales de control que servirían para establecer un sistema de calidad con resultados óptimos (Fink, Alberti y Mazziotta, 1997).

Nuestro país, lamentablemente, no participó del curso mencionado, no cuenta con programas para la evaluación externa de la calidad en hematología, ni con laboratorios certificados en la misma; pero, contrariamente casi no existe un laboratorio clínico que no realice hematología por la importancia de sus diagnósticos médicos basados en datos de la misma. Evidentemente, es necesario poner en práctica programas de evaluación externa de la calidad en hematología en todos los países latinoamericanos (Lewis y Burgess, 1969).

El aseguramiento de la calidad de Hematología mediante lineamientos del CLSI, están asegurando sistemas seguros de diagnóstico clínico, estas guías evalúan los procedimientos desde la colección de sangre relacionados al Tubo EDTA (ácido etilendiaminotetraacético) dipotásico $(\mathrm{K} 2)$ o tripotásico $(\mathrm{K} 3)$,el control de calidad en autoanalizadores, evaluación de resultados normales y anormales, el Delta check (guía CLSI H26-A2); hasta la morfología hematológica(CLSI H20-A2), la calidad del PCV (CLSI H07-A3) y la evaluación de sistemas automatizados para coagulación (CLSI H57-A2)(CLSI H26-A2, 2010; CLSI H20A2, 2007; CLSIH07-A3, 2000).

Dentro de la guía H26-A2, se menciona y esclarece el uso de las reglas de 3 , que indican la concentración aproximada de $\mathrm{Hb}$ y $\mathrm{Hto}$, a partir de multiplicaciones por 3 de RBC y $\mathrm{Hb}$, respectivamente. Cabe indicar que la variación esperada para este método es de $\pm 3 \%$, bajo ciertas condiciones como: que no exista alteraciones eritrocitarias, ni interferencias endógenas, como lipemia, bilirrubinemia, quilomicronemia, entre otras (CLSI H26-A2, 2010). Cierto es que, en nuestro país, la aplicación de este método tuvo que ver más con la facilidad económica y laboral. Por lo cual, el uso de esta regla se desarrolló masivamente en toda la nación, pero de manera inversa (partiendo de valores de Hto: Hto $\div 3=\mathrm{Hb}$ (mg/dl) y $\mathrm{Hb} \div 3=\mathrm{RBC}$ (millones)). Esta aplicación de la regla de 3 en nuestro país, tiene mucho tiempo en uso pero ningún estudio sobre variabilidad, discrepancias, limitaciones o error total (McKenzie, 2006). 
Como hipótesis planteamos que la evaluación continua del método de regla de 3 inversa según CLSI H26-A2, asegura las reglas internacionales de calidad. Por ello, el objetivo de la presente investigación fue determinar la imprecisión de parámetros eritrocitarios (Hematocrito, Hemoglobina y Recuento eritroide) con la regla de 3 según las guías del Instituto de Estándares Clínicos y de Laboratorio (CLSI) H26-A2, y discriminarlos por género

\section{MATERIAL Y MÉTODOS}

Con el fin de estudiar la imprecisión de los parámetros eritrocitarios (Hto, Hb y RBC) con la regla de 3 según las guías CLSI H26-A2,se realizó una investigación de tipo cuasi experimental cualitativa, de corte transversal prospectiva en el Laboratorio de Análisis Clínicos del Policlínico "La Fe" Urb. El Parral Lima 07. El tamaño muestral fue calculado usando EPIDAT 4.1 (Xunta de Galicia, España) considerando una sensibilidad de 0,95, una heterogeneidad de $50 \%$ y una precisión de 0,04 obteniéndose un tamaño muestral de 151 pacientes. Para tal fin se tomó una muestra de 170 pacientes derivados al servicio de hematología, provenientes de consultorios externos.

Las muestras incluidas en la investigación son las que cumplieron los criterios de la guía CLSI H26-A2 (pacientes sin patología eritrocitarios, ni interferencias endógenas notables, etc.).

\section{Procedimiento de Verificación y estandarización de microhematocrito:}

a. Evaluación de microcentrifuga: Para poder lograr y cumplir un empaquetamiento óptimo de células, se evaluó lo siguiente de acuerdo con la guía CLSI H07-A3:

- Radio mayor a 8,0cm.

- Capacidad de alcanzar la velocidad máxima en 30 segundos.

- Capacidad de sostener una Fuerza Centrífuga Relativa -RCF- de 10,000 a $15,000 \mathrm{~g}$ por al menos 5 minutos debajo de $45^{\circ} \mathrm{C}$.

- Calibración del reloj en intervalos de 30 segundos desde 0 hasta al menos 5 minutos.

- Verificación de la exactitud de reloj interno de la microcentrífuga con un cronómetro.

\section{b. Evaluación de rectitud de tubos capilares}

La gran cantidad de marcas de tubos capilares para microhematocrito cumplen con las especificaciones de la American Society for Testing and Materials (ASTM). Sin embargo, para verificar la calidad de los capilares y eliminar el potencial uso de capilares deformados se procedió a realizar lo siguiente.

- Medición de 12 capilares (6 sellados al lado "A" y 6 sellados al lado "B")

- Revisar que no existan diferencia sistemática entre valores hallados mediante la prueba de t-student. $(<2,571 \mathrm{TINV})$

Se justipreció y estandarizó la rectitud de tubos capilares para microhematocrito (t-student 2,198) del lote 20130909 de capilares no heparinizados de Biotech ${ }^{\mathrm{TM}}$ (Wicklow, Irlanda) según la guía CLSI H26-A2.

\section{Procesamiento de la muestra:}

Etapa pre analítica: La recolección se llevó a cabo en el área de Flebotomía, del Policlínico "La Fe" durante la mañana, en tubos BD Vacutainer ® (Franklin Lakes, Nueva Jersey) de $3 \mathrm{ml}$, tapa color lavanda con EDTA K2 y se mezcló por inversión (de 8 a 10 veces), de acuerdo con la guía CLSI H03-A6 (CLSI H26A2, 2010; CLSI H20-A2, 2007, CLSI H3-A6, 2007). El volumen de muestra en todos los casos fue mayor de $1 \mathrm{ml}$, ninguna presentó hemólisis, coágulos ni aglutinaciones.

Etapa analítica: Se procesaron las muestras inmediatamente y dentro de los 120 minutos respecto a la extracción sanguínea, con el fin de evitar falsos aumentos del tamaño de eritrocitos (Bain, Lewis y Bates, 2006). La determinación de microhematocrito manual se efectuó por el método de D'angelo y Lacombe, evaluado con la guía CLSI H07-A3 (Gonzáles, 2005). El cálculo de Hb y RBC derivados del valor de microhematocrito fue por las reglas de 3 inversas en cumplimiento de la guía H26-A2 (Bull et al., 2003).

La Regla de 3: Relaciona los parámetros concernientes a las células rojas. A saber, son 2 fórmulas:

$-3(\mathrm{RBC})=\mathrm{Hb} \mathrm{mg} / \mathrm{dl}$

$-3(\mathrm{Hb})=\mathrm{Hto} \%$

Las reglas de 3 inversas son:

${ }^{*} \mathrm{Hto} \div 3=\mathrm{Hb} \mathrm{mg} / \mathrm{dl}$

* $\mathrm{Hb} \div 3=\mathrm{RBC}$ millones. 
Se evaluó mediante microscopia, el frotis sanguíneo de todos los pacientes con el fin de descartar alteraciones eritrocitarias y hemopatías. El conteo de WBC se realizó con la cámara de Neubauer por el método hematimétrico, corroborado con la concentración de la capa flogística del microhematocrito, donde no se hallaron alteraciones.

Etapa post analítica: Los resultados fueron digitados en el libro de reportes del servicio, previa validación del personal de laboratorio y entregados a los pacientes en los tiempos estipulados.

Técnica de recolección de datos: Los datos se registraron en un instrumento de hoja de cálculo de Microsoft Office Excel 2010 para Windows, desde el libro de reportes del área. Dentro de la matriz de calidad se utilizó la guía del CLSI H26-A2, para evaluar valores clásicos de variabilidad (desviación media, varianza muestral, desviación estándar y coeficiente de variación de Pearson -\%CV-) y compararlos con estudios sobre análisis de imprecisión realizados con anterioridad, principalmente con los descritos por el CLSI por ser estos protocolos internacionales estandarizados de evaluación que definen y protocolizan la recolección de datos, el procesamiento analítico y estadísticos de los mismos y la interpretación de los resultados obtenidos (CLSI H26-A2, 2010, ISPC, 2009).

Análisis estadístico: El análisis estadístico fue mediante SPSS versión 20.0 donde se utilizó t-de student para la evaluación de rectitud de tubos capilares.

La principal limitación fue que la regla de 3 empleada no fue la adscrita según la guía CLSI H26-A2, la utilizada en la investigación fue la razón entre el Hto $\div 3=\mathrm{Hb}$ y $\mathrm{Hb} \div 3=\mathrm{RBC}$, empleada comúnmente en los laboratorio hematológicos del país. Pese a esta limitación, somos los pioneros en acometer la tarea de esclarecer la impresión existente en la determinación de parámetros eritrocitarios con la regla de 3 inversa, usada en gran cuantía en la comunidad.

Ética: la información obtenida se mantendrá en anonimato y sólo se utilizará para esta investigación.

\section{RESULTADOS}

De 170 muestras de pacientes evaluados mediante microhematocrito manual, 60 fueron varones, 102 mujeres y 8 eliminados. El \% CV de Hto, $\mathrm{Hb}$ y RBC fueron $1,9 \%, 5,6 \%$ y $16,9 \%$, respectivamente. La proporción de imprecisión discriminada por género se muestra a continuación en la Tabla 1.

Tabla 1. Imprecisión discriminada por género( $n=162)$

\begin{tabular}{lrr}
\hline $\begin{array}{l}\text { Parámetros } \\
\text { eritrocitarios }\end{array}$ & Varones & Mujeres \\
\hline Hto & $2,36 \%$ & $2,83 \%$ \\
$\mathrm{Hb}$ & $7,80 \%$ & $8,56 \%$ \\
$\mathrm{RBC}$ & $23,50 \%$ & $26,21 \%$ \\
\hline
\end{tabular}

Los resultados del estudio en contraposición con valores de requerimientos médicos para el manejo del paciente según The Clinical Laboratory Improvement Amendments 1988-CLIA '88 -, la Sociedad médica japonesa y valores de manejo de pacientes (Patient management) concernientes en la guía CLSI H26-A2, se muestran en la Tabla 2.

Los requerimientos médicos (cambios seriales en un paciente expresados en \% CV individual, necesarios para el manejo clínico del paciente) necesita una imprecisión más corta, a tal punto de no parecer imprecisión de laboratorio, sino variabilidad biológica.

Tabla 2. Imprecisión interindividual y requerimientos médicos del manejo de paciente.

\begin{tabular}{ccccc}
\hline $\begin{array}{l}\text { Parámetros } \\
\text { eritrocitarios }\end{array}$ & $\begin{array}{l}\text { Valores del } \\
\text { estudio }\end{array}$ & CLIA '88 & $\begin{array}{l}\text { Japanese Medial } \\
\text { Association * }\end{array}$ & $\begin{array}{c}\text { Patient } \\
\text { management * }\end{array}$ \\
\hline Hto & $1,9 \%$ & 3 & - & $1,6 \%$ \\
Hb & $5,6 \%$ & $3,5 \%$ & 3 & $1,4 \%$ \\
RBC & 16,9 & 3 & 4 & $1,4 \%$ \\
\hline
\end{tabular}

*Estudios descritos en la guía CLSI H26-A2 
A la par, en la Tabla 3 se muestran los valores comparables entre 2 estudios sobre imprecisión potencial relacionados a parámetros eritrocitarios de $\mathrm{Hb}$ y $\mathrm{RBC}$ (expresados en rangos) mencionados en la guía CLSI H26-A2, (el grupo italiano "State of the art" y otros autores que consideraron $\leq 0,5$ de variabilidad biológica para la evaluación intraensayo). La matriz de correlaciones entre variables resultó adecuada $(p=<0,05)$

Tabla 3. Imprecisión intraensayo tradicional en parámetro eritrocitarios.

\begin{tabular}{cccc}
\hline $\begin{array}{l}\text { Parámetros } \\
\text { eritrocitarios }\end{array}$ & $\begin{array}{l}\text { Valores del } \\
\text { estudio }\end{array}$ & State of the art * & $\begin{array}{c}\leq 0,5 \text { within subject } \\
\text { biological variation * }\end{array}$ \\
\hline Hto & $1,8 \%$ & - & - \\
Hb & $5,6 \%$ & $0,6-1,2 \%$ & $0,8 \%-1,5 \%$ \\
RBC & $16,9 \%$ & $0,5-1,3 \%$ & $0,8 \%-1,7 \%$ \\
\hline
\end{tabular}

*Estudios descritos en la guía CLSI H26-A2

\section{DISCUSIÓN}

El cuadro hemático es una de las pruebas que más se solicita al laboratorio clínico y es la prueba de laboratorio que más aporta al clínico en la evaluación de un paciente. Este conjunto de exámenes, que evalúan distintos elementos sanguíneos, están compuestos de diferentes parámetros según el tipo de hemograma, cada uno con un coeficiente de variación distinto, pero que en su mayoría no exceden el $3 \%$ (Campuzano, 2007).

La evaluación realizada demostró, con excepción del Hto que tiene precisión y exactitud adecuada y aceptable para la práctica clínica, que los resultados de imprecisión expresados están fuera del rango máximo permisible de imprecisión intraensayo y son incongruentes con los límites de máxima variación establecidos por los requerimientos médicos según la guía CLSI H26-A2, por lo que resulta poco útil para el cálculo de $\mathrm{Hb}$ y $\mathrm{RBC}$ (WHO, 2000).

El \% CV de Hto $(1,9 \%)$ se encontró dentro de la imprecisión máxima permisible establecido por CLIA '88, debido principalmente a la correcta verificación y estandarización de equipos e insumos (microcentrífuga y tubos capilares) y al cumplimiento de los lineamientos internacionales de calidad (D'angelo y Lacombe bajo la guía CLSI H07-A3) que aseguran la calidad final de los resultados y mejora la confiabilidad de resultados emitidos por el laboratorio clínico hematológico. (Gonzáles, 2005; CLSI H07-A3, 2000).

Las derivaciones a partir de Hto son incongruentes con los límites de máxima variación establecidos, tanto por los requerimientos médicos de la fuente japonesa, CLIA '88 y los requerimientos médicos para el manejo del paciente. Estos valores de $\mathrm{Hb}$ y RBC tienen una diferencia muy significativa, conforme se calcula el siguiente valor a partir de la razón matemática anterior $(5,6 \%$ para $\mathrm{Hb}$, $16,9 \%$ para $\mathrm{RBC}$ ). Los valores comparados en la Tabla 2, demuestran que la técnica de microhematocrito manual para determinación de parámetros eritrocitarios con las reglas de 3 usadas para el manejo médico es crítico, al derivarse de esta el valor de $\mathrm{Hb}$ y RBC. Tanto para CLIA '88 como para la asociación médica japonesa los valores de $\mathrm{Hb}$ son rígidos y no se desvían más de $3,5 \%$, en nuestra investigación, demostramos que el uso de la regla de 3 inversa para la determinación de los parámetros eritrocitarios resulta poco útil para el cálculo de Hb y RBC (CLSI H26-A2, 2010; CLSI H07-A3, 2000).

Estos valores resultantes de una imprecisión constante, no pueden usarse para derivar valores hematimétricos (volumen corpuscular medio -VCM-, hemoglobina corpuscular media -HCM-, y la concentración de hemoglobina corpuscular media-CHCM-), ya que, los valores ecuacionales de donde derivan están fuera del rango máximo permisible de imprecisión, entonces, estos expresarían valores indeterminados.

De la misma forma, la discriminación por género demostró que la mayor imprecisión está en mujeres en comparación de los hombres, así, en ambos casos se obtuvieron resultados que superaron el máximo de imprecisión permisible (con excepción de CLIA'88 para Hto). Si bien, esto podría estar influenciado por 
la cantidad muestral (100 pacientes mujeres frente a 55 varones), aunque este fenómeno se observa constantemente en las estimaciones de Desviación estándar y no en Coeficientes de Variación de Pearson; consideramos esta evaluación superlativa entorno a la valoración de calidad del proceso (CLSI H26-A2, 2010; CLSI H07-A3, 2000).

Se determinó la imprecisión intraensayo tradicional, basados en estudios hematológicos por el grupo italiano "State of the art", de todos los intervalos de los parámetros eritrocitarios, estableciéndose los valores mostrados en la Tabla 3. Los intervalos del grupo italiano como los descritos por diversos autores, que establecen $\leq 0,5 \%$ de variación biológica intraindividual, fijan los valores máximos para $\mathrm{Hb}$ y $\mathrm{RBC}$. Es de notar que lo hallado en nuestra investigación difiere total y alarmantemente.

El gran uso de estas reglas de 3 inversas en la comunidad, se debe en gran medida a un desconocimiento del impacto clínico, a la facilidad $y / 0$ urgencia en los laboratorios clínicos nuevos, los limitados en tecnología o con intereses de lucro (Campuzano, 2007). El problema de estas estimaciones, es que hasta ahora no se pueden considerar resueltos; esto lo comprueban nuestros resultados en base a los cuales se debe desarrollar evaluaciones de mayor cuantía, duración y alcance.

Finalmente, sugerimos realizar la regla de 3 convencional, como lo menciona el CLSI, que debe de someterse a evaluación y estandarización de procesos: dependiendo de los equipos (microcentrífuga, espectrofotómetros), instrumentos (tubos capilares, cartilla de lectura estandarizada), registros (análisis de datos para establecer precisión del ensayo por cada servicio, establecer un POE (procedimiento operacional estandarizado), ubicación geográfica (latitudes, metros sobre el nivel del mar), frecuencia del tipo pacientes (grupo etario, pacientes pediátricos, gestantes); y al cumplimiento de los requisitos de calidad que ofrecen las variabilidad biológica en base al error aleatorio, sistemático o total establecidos mediante estudios y reportes dentro de un sistema de aseguramiento integral de la calidad. Sugerimos también, corregir y/o convertir los valores del hematocrito manual mediante la fórmula de Perkins o descontinuar su uso.
En eso contexto, la evaluación de métodos es clave para el cumplimiento de los requerimientos internacionales de calidad, organismos de acreditación y para la mejora continua.

\section{REFERENCIAS BIBLIOGRÁFICAS}

Bain, BJ., Lewis, S. \& Bates, I. (2006) Basic haematological techniques. In Lewis SM, Bain BJ, Bates I. (Ed) Dacie and Lewis practical haematology, (5th), Edinburgh ; New York: Churchill Livingstone, 26-57.

Bull, B., Fujimoto, K., Houwen, B., Klee, G., van Hove, L., van Assendelft, W., Bunyaratvej, A., Buttarello, M., Davis, B., Koepke, J., Lewis, S., Machin, S., d'Onofrio, G., Rowan, R. \& Tatsumi, N. (2003). International Council for Standardization in Haematology (ICSH) recommendations for "surrogate reference" method for the packed cell volume. Lab Hematol, 9, 1-9.

Campuzano, G. (2007). Del hemograma manual al hemograma de cuarta generación. Medicina \& Laboratorio, 13: 511-550.

Clinical and Laboratory Standards Institute (2007). Procedures for the collection of Diagnosis Blood Specimens by Venipuncture. Pennsylvania: H3-A6, 27(26). Wayne, Pensilvania: CLSI document.

Clinical and Laboratory Standards Institute (2000).Procedure for Determining Packed Cell Volume by the Microhematocrit Method (Third Edition). H07-A3, 30(14). Wayne, Pensilvania: CLSI document.

Clinical and Laboratory Standards Institute (2007). Reference Leukocyte (WBC) Differential Count (Proportional) and Evaluation of Instrumental Methods; Approved Standard (Second Edition). $\mathrm{H} 20-\mathrm{A} 2$, 27(4).

Clinical and Laboratory Standards Institute (2010).Validation, Verification, and Quality Assurance of Automated Hematology Analyzers (Second edition).H26-A2, 30(14). 
Cooper, N., Hart, D., Bates, I., \& Cotter, F. (2011).The importance of developing world hematology. Brit J Haematology, 154(6), 667.

Fink, N., Alberti, A. \& Mazziotta, D. (1997). Evaluación externa de la calidad analítica en hematología: una necesidad en América Latina. Public Health, 2(3),181-188.

Gonzáles, J. (2005). Técnicas y métodos de laboratorio clínico. (2da ed.) Barcelona: Masson, S.A.

Instituto de Salud Pública de Chile (ISPC) (2009). Guía técnica normalizada de Control de Calidad. ISP-CC-03/2009. Departamento de Laboratorio Biomédico. Gobierno de Chile, Ministerio de Salud. Ñuñoa: ISPC Publisher.

Lewis, S. \& Burgess, B. (1969). Quality in haematology: report of interlaboratory trials in Britain. BrMed J, 2, 253-256.
McKenzie, S. (2006). Hematología Clínica. (2da edición). México D.F.: Manual Moderno.

Nevalainen, D., Berte, L., Kraft, C., Leigh, E., Picaso, L.. \& Morgan, T. (2000). Evaluating Laboratory Performance on Quality Indicators with the Six Sigma Scale. Archives of Pathology \& Laboratory Medicine, 124(4), 516-519.

World Health Organization (WHO) (2000).Recommended method for the determination of packed cell volume by centrifugation (prepared by Expert Pane on cytometry of the International Council for WHO/DIL/00.WHO, Geneva; 3-7

Correo electrónico: jeelms@outlook.com Corrección de pares:

Recibido: 19-03-2015

Aceptado:20-06-2015 